\title{
$A b$ initio investigation of the atomic volume, thermal expansion, and formation energy of WTi solid solutions
}

\author{
R. Bodlos $\odot,{ }^{1}$ T. Dengg, ${ }^{1}$ A. V. Ruban, ${ }^{1,2}$ M. Dehghani $\odot,{ }^{1}$ L. Romaner, ${ }^{1}$ and J. Spitaler ${ }^{1}$ \\ ${ }^{1}$ Materials Center Leoben Forschung GmbH, Roseggerstrasse 12, 8700 Leoben, Austria \\ ${ }^{2}$ Department of Materials Science and Engineering, KTH Royal Institute of Technology, 10044 Stockholm, Sweden
}

(Received 22 December 2020; revised 1 March 2021; accepted 16 March 2021; published 1 April 2021)

\begin{abstract}
WTi is used as an adhesive layer in integrated circuit devices. The temperature dependent mechanical properties of WTi are still largely unexplored. In this paper we investigate WTi solid solutions with density functional theory calculations to determine the temperature and concentration dependent behavior of volume and coefficient of thermal expansion. The coefficient of thermal expansion is analyzed in terms of the bulk modulus, heat capacity, and Grüneisen parameter. Furthermore, we gain insight into the bonding of the system via investigation of the electronic structure, phonon density of states, and analysis of the formation energy. Low Ti concentrations lead to strong W-Ti bonding, as manifested in additional high frequency peaks in the phonon density of states. As a consequence, deviations from Vegard's law are found at low Ti concentrations, with a minimum of the lattice constant at about 15 at. \% Ti. The CTE as a function of Ti concentration shows a negative trend at low temperatures and Ti concentrations, which is related to a strong decrease of heat capacity. Finally we show that the Debye-Grüneisen model yields results for WTi comparable to the quasiharmonic approach at a fraction of the computational cost.
\end{abstract}

DOI: 10.1103/PhysRevMaterials.5.043601

\section{INTRODUCTION}

Metallizations in integrated circuits (ICs) have been a subject of intense research. As the performance of the IC is limited by the resistivity of its metallization, only four elements can be used: $\mathrm{Au}, \mathrm{Ag}, \mathrm{Al}$, and $\mathrm{Cu}$. $\mathrm{Ag}$ and $\mathrm{Au}$ are problematic due to their high diffusion rate in dielectrics and are therefore not suited for most IC applications. Al, which used to be the standard material for metallization, offers acceptable resistivity at a low density. However, it is inferior to the other three elements in terms of resistivity and leads to pronounced electromigration, a diffusion process that can lead to the failure of the electronic devices. This has mainly been solved by alloying $\mathrm{Al}$ with $\mathrm{Cu}$, which slows down the electromigration rate. Nevertheless the increasing demand for low resistivity has caused $\mathrm{Cu}$ to replace $\mathrm{Al}$ and $\mathrm{Al}-\mathrm{Cu}$ as a metallization for IC devices [1-3]. A challenge when using $\mathrm{Cu}$ is its limited adhesion to the dielectric layer of the IC device which in turn leads to delamination [4-6]. An interlayer is needed to facilitate the adhesion of the $\mathrm{Cu}$ layer to the dielectric layer. Physical vapour deposition sputtered WTi is a commonly used interlayer, as it shows good adhesion and high thermal stability in a wide range of concentrations. As a consequence, most experimental publications focus on the

Published by the American Physical Society under the terms of the Creative Commons Attribution 4.0 International license. Further distribution of this work must maintain attribution to the author(s) and the published article's title, journal citation, and DOI. sputtering process and the temperature dependence of barrier properties [7-12].

In terms of $a b$ initio calculations, a series of articles dealing with the physical and thermodynamic properties of $\mathrm{W}$ alloys as a function of alloy concentration were published. They were based on ordered structures [13], special quasirandom structures (SQSs) [13-16], the cluster expansion approach [17-20], the virtual crystal approximation (VCA) [21-23], and the coherent potential approximation (CPA) $[24,25]$. WTi, however, has remained relatively unexplored. One work [13] has investigated elastic properties, atomic volume, and formation energies of ordered WTi structures for assessing mechanical properties. Furthermore, a recent work by Ångqvist et al. [26] deals with ab initio calculations of WTi in the entire concentration range with a focus on the formation energy and revision of the phase diagram.

Despite the high relevance of WTi as a diffusion barrier, many fundamental physical properties of the solid solution are still not known. The solid solution is not only the main phase at high temperatures according to the phase diagram of WTi [27], which is valid for the case of thermodynamic equilibrium. It also results from process conditions starting from a random distribution of atoms where the temperature is too low or cooling is too fast to allow one to achieve thermodynamic equilibrium via diffusion. This is the case for typical sputtering processes used in microelectronics, as well as metallurgical processes involving quenching from high temperatures. Several publications confirm the sputtered WTi as a solid solution phase $[28,29]$. In particular this includes the dependence of the atomic volume on composition over the whole Ti concentration range. The latter is highly relevant in view of the decomposition observed in $\mathrm{WTi}^{12}$ which can 
cause volumetric mismatch. Furthermore, phonon properties and thermal expansion, which are relevant for residual stress minimization during operation of the ICs, has so far not been investigated systematically. This work closes this gap with a systematic investigation on the concentration-dependent lattice constants and formation energies at $0 \mathrm{~K}$ together with the temperature-dependent atomic volume and coefficient of thermal expansion (CTE) of the WTi solid solution. These properties are highly relevant for understanding and predicting the mechanical properties in layered systems used in ICs.

\section{METHODOLOGY}

Density functional theory (DFT) calculations were performed using the Vienna Ab initio Simulation Package (VASP) and the projector augmented wave (PAW) method [30-37], with exchange-correlation effects treated in the PerdewBurke-Ernzerhof (PBE) approximation [37]. For all calculations of cubic systems, a $k$-point density corresponding to $20 \times 20 \times 20 k$ points in the conventional two-atom unit cell was used, while for the two-atom hcp Ti cell, $22 \times 22 \times 14$ $k$ points were employed. In all cases the $k$-point mesh was Gamma centered. W was treated using 12 valence electrons $\left(5 p^{6} 5 d^{4} 6 s\right.$ [2]) and $\mathrm{Ti}$ using ten valence electrons $\left(3 p^{6} 3 d^{2} 4 s\right.$ [2]), respectively, and the energy cutoff was set to $400 \mathrm{eV}$.

In order to model a solid solution a random distribution of the alloying elements on the crystal lattice needs to be simulated. This was done using 16- and 128-atom supercells with minimized Warren-Cowley short range order (SRO) parameters for the first five coordination shells [38-40] for $6.25 \%$, $12.5 \%, 18.75 \%, 25 \%, 31.25 \%, 50 \%, 68.75 \%, 75 \%, 81.25 \%$, $87.5 \%$, and $93.75 \%$ of titanium atoms. See the Supplemental Material [41] for the Warren-Cowley SRO parameter for all supercells. The 128-atom supercell proved to be an excellent approximation to an ideal solid solution, with all SRO parameters up to the fifth coordination cell very close or equal to zero. These supercells were used to calculate formation energies and served as a benchmark for the 16-atom supercells in terms of energetics. The calculation of all other properties were based on the 16-atom supercells.

The equilibrium volume for each Ti concentration was determined by fitting the Birch-Murnaghan [42] equation to the DFT total energy results, where nine different volumes were considered. In the total energy calculations, atomic positions were allowed to relax. The temperature dependent volume and linear thermal expansion coefficient were calculated using two different methods: the Debye-Grüneisen (DG) model as is implemented in THERMELPY [23], and from the quasiharmonic approximation (QHA) from explicit phonon calculations using PHONOPY [43].

The DG model applied in this work follows the methodology described in Refs. [23,44,45]. The volume at a given temperature is obtained from minimizing the Helmholtz free energy. The latter is obtained from the Grüneisen parameter $\gamma$ as described in Ref. [46] The temperature-dependent Grüneisen parameter $\gamma(T)$ is obtained via

$$
\gamma(T)=\gamma_{\mathrm{LT}}+\frac{\frac{1}{3} f}{1+f} \text { with } f=e^{-\left(\Theta_{D}^{0} / 3 T\right)^{3}},
$$

where $\Theta_{D}^{0}$ is the Debye temperature at the $0-\mathrm{K}$ equilibrium volume which we calculate as described by Korzhavyi et al. (Ref. [47]) and $\gamma_{\mathrm{LT}}$ is the low temperature limit of the Grüneisen parameter which is calculated as described in Ref. [46]. Note that this particular form of $\gamma(T)$ is similar to that in Ref. [48]. Although it has not been reported, it was already used in Refs. [45,47]. Compared to the other implementations of the DG model discussed before [46-48] it yields the best agreement to QHA calculations reported below.

In the QHA calculations, the small displacement method was used to determine the force constant matrix for a $2 \times 2 \times 2$ supercell of the 16 -atom SQS (128 atoms in total). The phonon frequencies, the phonon density of states, and the vibrational free energy were obtained by the PHONOPY code [49]. In addition, the accuracy of forces was improved by using an additional support grid for the evaluation of the augmentation charges (ADDGRID = TRUE) [50,51]. The vibrational free energy was combined with the equation of state (EOS) for $0 \mathrm{~K}$ to obtain the free energy as a function of volume for each temperature and therefore the temperature dependent equilibrium volume. The linear coefficient of thermal expansion (CTE) $\alpha$ was obtained from numeric differentiation of the volume as a function of $T$.

We have also done calculations by the exact muffin tin orbital method within the coherent potential approximation (EMTO-CPA) [52-55] for electronic structure of random alloys. The Lyngby version of the EMTO code has been used. In these calculations $\mathrm{Ti} p$-semicore states and $\mathrm{W} f$ - and $p$ semicore states have been included. A $34 \times 34 \times 34 k$-point mesh of the Monkhorst-Pack grid was used in the calculations and the total energy was obtained within the full charged density technique using the PBE exchange-correlation energy.

\section{RESULTS}

\section{A. Electronic density of states}

In this work we target a fundamental understanding of the WTi solid solution. For this purpose it is useful to first investigate what impact alloying has on the occupation of electronic states. In the upper panel of Fig. 1, we show the electronic density of states (DOS) as a function of $\mathrm{Ti}$ concentration obtained with EMTO-CPA (the corresponding VASP supercell results are included in the Supplemental Material [41]). The most important feature of the DOS for a bcc crystal is a shallow valley around the Fermi energy, forming the so-called pseudogap [56-58]. For the Ti concentrations up to $31.25 \%$, the shape of the DOS changes rather little. The main change is in the position of the Fermi energy, which shifts to lower energies with Ti concentration since Ti has only two electrons in the $3 d$ shell, while $\mathrm{W}$ has four electrons in this shell. In the lower panel of Fig. 1, the electronic DOS as obtained for pure W with VASP is shown, together with the shift of the Fermi level with titanium concentration due to the change in the number of valence electrons, based on a rigid-band approach. One can clearly see that in this case the Fermi level remains inside the pseudogap until $12.75 \% \mathrm{Ti}$, and with increasing $\mathrm{Ti}$ concentration it starts to move to larger values of the DOS. The smearing and broadening of the DOS peaks with Ti concentration is due to the randomness captured by the 

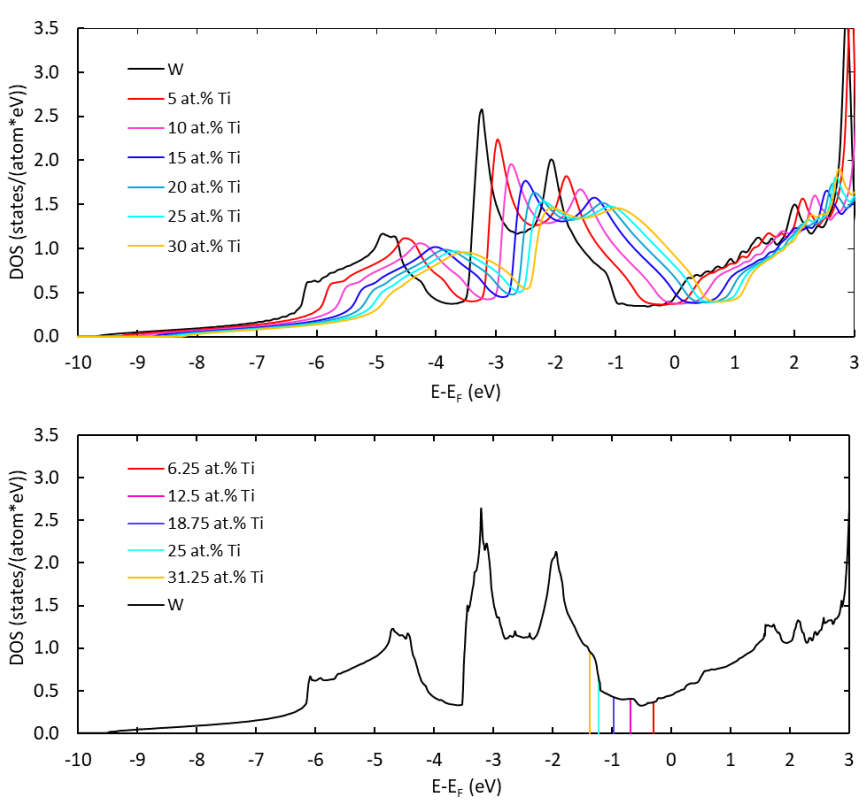

FIG. 1. Top: Electronic DOS for WTi random alloys obtained by the EMTO-CPA method showing the overall shift of the DOS with Ti concentration. Bottom: Electronic DOS for pure W obtained by VASP; the colored vertical lines indicate the position of the Fermi level using the DOS of $\mathrm{W}$ as a basis, but changing the filling of the $d$ bands according to the Ti concentration.

CPA, whose effect increases with concentration. The change in DOS and, in particular, the shift of the Fermi level is the main cause for changes in lattice parameters, phonon energies, and coefficient of thermal expansion discussed in the later sections.

\section{B. Atomic volume as a function of Ti content}

As a first step we shortly focus on the volumes of pure $\mathrm{W}$ and Ti crystals. Our results, shown in Fig. 2, show that the atomic volume of $\mathrm{Ti}$ is larger than the one of $\mathrm{W}$. Our

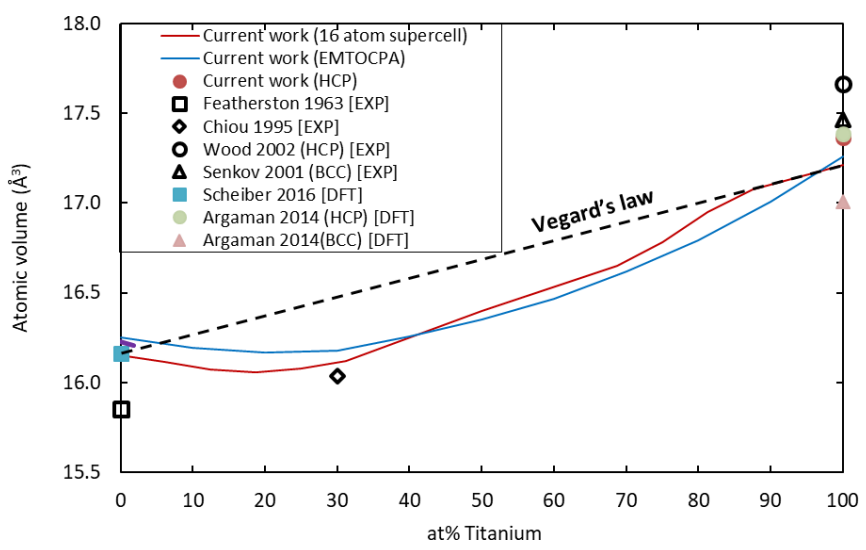

FIG. 2. Atomic volume vs Ti concentration for bcc WTi: Lines denote results from the current work; filled symbols the current result for hcp Ti and DFT results from literature [59,60]; open symbols show experimental results at room temperature $[7,61,62,64]$. The black dashed line indicates the volume as a function of concentration according to Vegard's law. results closely match previous $a b$ initio literature data for pure W [59] and pure Ti [60], respectively, except for the result for bcc Ti from Argaman et al. [60], where a different exchange-correlation potential was used. Also, the results are in good agreement with experiment. The DFT results with PBE slightly overestimate the volume for $\mathrm{W}$ but underestimate the hcp Ti volume when compared to the experimental data [61,62], which is a known feature of the potential $[60,63]$. For bcc $\mathrm{Ti}$ an extrapolation to room temperature is available [64] and shows the same trend as with hcp Ti. Overall, the larger volume of $\mathrm{Ti}$ with respect to the $\mathrm{W}$ is also observed in experiment.

In Fig. 2, the volume per atom of $\mathrm{W}_{1-x} \mathrm{Ti}_{x}$ as a function of Ti concentration $x, V(x)$, is shown. For both VASP and EMTO-CPA calculations, $V(x)$ exhibits a nonlinear behavior with a decrease for small concentrations and an increase for concentrations larger than 20 at. \% Ti. The decrease of volume for small $\mathrm{Ti}$ concentrations seems counterintuitive, since $\mathrm{Ti}$ has a larger atomic volume than $\mathrm{W}$. This nonlinear behavior was also observed previously in the calculations by $\mathrm{Hu}$ et al. [65], where it was discussed only for low Ti concentrations. A nonlinear trend is also observed in experiment when comparing results for $\mathrm{W}, \mathrm{W}_{0.7} \mathrm{Ti}_{0.3}$, and hcp $\mathrm{Ti}[7,61,62]$.

The nonlinearity marks a deviation from Vegard's law $[66,67]$ which is not unexpected considering that $\mathrm{W}$ and $\mathrm{Ti}$ are relatively dissimilar atoms with rather different valence electron number, cohesive energy, and electron density [68]. MoTi, a system similar to WTi, shows a similar deviation from Vegard's law according to first principles calculations by Barzilai et al. [69] and Sahara et al. [70]. We note that the minimum in the volume vs concentration curve correlates with the point where the Fermi level moves out of the pseudogap in the DOS and starts to climb the shoulder of the peak located below the pseudogap.

\section{Formation energy}

The formation energy of $\mathrm{W}_{1-x} \mathrm{Ti}_{x}$ as a function of $\mathrm{Ti}$ concentration $x$ is obtained from the energy (per atom) of the solid solution, $E(x)$, with respect to the energies of pure tungsten and titanium as

$$
E_{\mathrm{Form}}(x)=E(x)-\left[E_{\mathrm{Ti}}^{\mathrm{bcc} *} x+E_{\mathrm{W}}^{\mathrm{bcc} *}(1-x)\right] .
$$

Figure 3 shows the formation energy of bcc $\mathrm{W}_{1-x} \mathrm{Ti}_{x}$, $E_{\text {Form }}(x)$, using bcc titanium as reference, while the dashed black line connects the true low-temperature ground states of the pure phases, i.e., bcc $\mathrm{W}$ and hcp Ti. Phases below the dashed line are expected to be stable at low temperatures, while phases above the dashed line are expected to be unstable with respect to demixing. In order to understand the impact of different approximations on the result, we show EMTO-CPA (blue line) results together with those of VASP supercells without relaxation (light green and orange line) and with relaxation (dark green and orange). The EMTO-CPA calculations do not include local lattice relaxation effects and their results are in good agreement with the corresponding VASP calculations. At the same time our VASP calculations with local relaxations are in good agreement with the results obtained from the effective cluster interactions (ECIs) by Ångqvist et al. [26]. The curve was calculated from the list of effective clusters [71] via the 


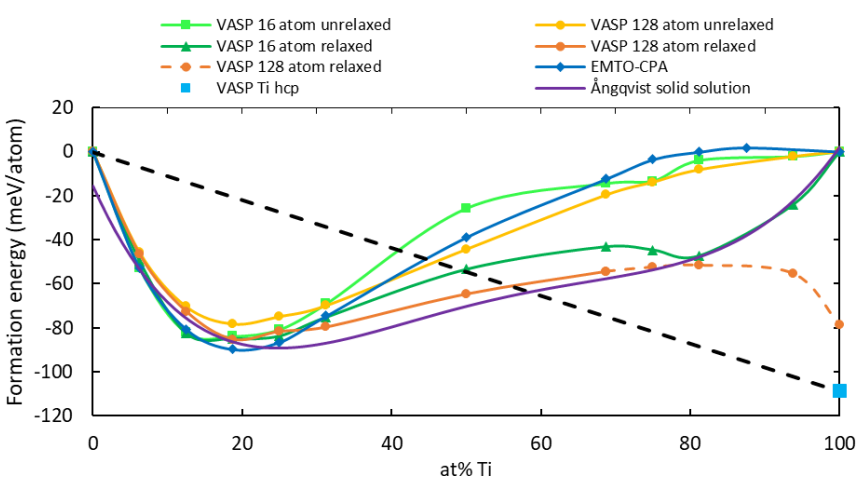

FIG. 3. Formation energy of bcc $\mathrm{W}_{1-x} \mathrm{Ti}_{x}$ from different approaches: EMTO-CPA calculation without relaxation (blue line), VASP result for 16-atom supercell (light green line) and 128-atom supercell without relaxation (light orange line), and VASP results for the 16-atom supercell (dark green line) and 128-atom supercell with relaxations (dark orange line). The dashed orange line represents the energies of 128-atom supercell for large Ti concentrations where large atomic displacements from the ideal bcc positions took place. The violet line is based on the effective cluster interactions fitted to DFT calculations (Ångqvist et al. [26]). See text for more details.

equation $E_{\mathrm{Form}}=\sum_{\alpha} m_{\alpha} J_{\alpha}(2 x-1)^{n}$ [72], where $m_{\alpha}$ is the multiplicity of the cluster $\alpha, J_{\alpha}$ is the ECI of the cluster, $n$ is the degree of the cluster, and $x$ is the concentration of Ti.

Let us now look at the effect of supercell size on the formation energy, comparing the results of 16-atom and 128-atom supercell calculations. For low Ti concentrations, the 16-atom supercell yielded formation energies in good agreement with the results for the 128-atom supercell, with differences smaller than $3.1 \mathrm{meV} /$ atom. For Ti concentrations larger than $12.5 \%$, however, the difference gets significantly larger. We attribute this behavior to the increasing tendency of the atoms to move off-center from their ideal positions and to form a local coordination different from the bcc one, which is possible only in the 128-atom supercell, while the 16-atom cell severely limits the possible atomic rearrangements due to the limited cell size. In the case of the $93.75 \%$ Ti the maximum displacement in the 16-atom cell $(0.13 \AA)$ is smaller by a factor of 4 compared to the 128 -atom cell $(0.53 \AA)$. As a consequence the 16 -atom supercell calculations follow the trend obtained by the cluster expansion method which is also based on the total energies of small supercells (up to 12 atoms). In contrast the 128-atom supercell results show strong deviations from "bcc-like" behavior, as seen in the dashed orange line in Fig. 3. In fact we found that the atomic relaxations were so large that the local coordination analysis (common neighbor analysis) [73-75] did not assign atomic sites in the supercell as belonging to the bcc crystal structure anymore. Note that Lieser et al. [15] found similar deviations for the HfW system. As seen in the final point on the dashed orange line of Fig. 3, we also investigated a 128-atom bcc supercell for pure Ti, but starting with atomic positions shifted away from the high symmetry positions based on the relaxed $93.75 \%$ Ti supercell. We again obtained large relaxations and a much lower energy than the one for ideal bcc Ti.

The solid solution is energetically favorable up to about $55 \% \mathrm{Ti}$, as found below the dashed line in this concentration

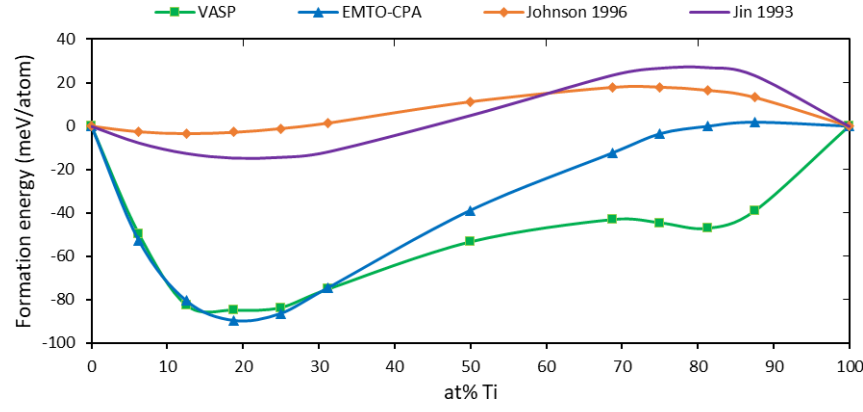

FIG. 4. Formation energies derived from Redlich-Kister parameters of CALPHAD databases (orange and purple) compared to our VASP formation energy results from the 16 atom SQS (light green) and our EMTO-CPA (dark blue) calculations.

range. At $\mathrm{Ti}$ concentrations larger than 20\%, however, the unrelaxed results differ significantly from the relaxed ones. This energy gain obtained from atomic relaxations indicates a gradual decrease in stability with increasing Ti concentration, leading to stronger local relaxations. Indeed pure bcc Ti is known to be mechanically unstable [76].

We do not consider here the hcp phase of WTi as it is not found in the phase diagram and the energy difference between the bcc and the hcp phase of pure $\mathrm{W}, \Delta E^{\mathrm{bcc}-\mathrm{hcp}}$, is as large as $-499 \mathrm{meV} /$ atom. This energy difference is significantly higher than $\Delta E^{\text {bcc-hcp }}$ for hcp Ti $(109 \mathrm{meV})$, which means that the concentration range in which hcp WTi is stable is either very narrow or does not exist at all. Calculations by Giret et al. confirm a huge gap between bcc W and other possible modifications such as hcp [77]. Furthermore Ångqvist et al. [26] calculated hcp WTi but as expected only found positive formation energies for the entire concentration range.

Our results obtained for the formation energy can be compared to well-known and frequently used expressions from CALPHAD databases of Johnson [78] and Jin [79]. We used the the Redlich-Kister parameters [80] reported in those databases to generate the corresponding formation energies at $0 \mathrm{~K}$ as seen in Fig. 4. The parameters are reproduced in Table I. For completeness and better reusability of our results the parameters obtained by fitting the Redlich Kister polynomial to the ab initio formation energies are also reported in the table. The CALPHAD assessments differ significantly from our VASP and EMTO-CPA results. While there exists a general consensus that the formation energy should be lower in the lower concentration range compared to the higher concentration range, the quantitative predictions differ substantially. Our results indicate the necessity for reassessment of the WTi thermodynamic database taking into account that formation energies at low temperature should be much more negative as currently assumed. This might lead to changes in phase boundaries in the phase diagram especially in the temperature range below $1000 \mathrm{~K}$ as already indicated by Angqvist et al. [26].

\section{Phonon density of states}

The phonon density of states (PDOS) of bcc WTi as a function of Ti concentration is plotted in Fig. 5. It shows remarkable features: First, the PDOS of pure bcc W and the 
TABLE I. Redlich-Kister parameters based on our formation energy curves and from CALPHAD calculations [78,79]. The VASP and EMTO-CPA values have no $T$ dependence and are only valid for $0 \mathrm{~K}$.

\begin{tabular}{lrrrr}
\hline \hline & \multicolumn{1}{c}{ VASP } & EMTO-CPA & Johnson [72] & Jin [73] \\
\hline$L 0(\mathrm{meV})$ & -199.41 & -151.77 & $44.70+0.13 * \mathrm{~T}$ & $19.81+0.11 * \mathrm{~T}$ \\
$L 1(\mathrm{meV})$ & 211.50 & 491.15 & $101.24-0.04 * \mathrm{~T}$ & $218.80-0.09 * \mathrm{~T}$ \\
$L 2(\mathrm{meV})$ & -590.35 & -388.33 & 0 & 53.64 \\
\hline \hline
\end{tabular}

real modes of bcc $\mathrm{Ti}$ almost perfectly overlap in terms of the occupied frequency range, in agreement with $a b$ initio calculations [23,81]. Moreover, experimental results of the phonon DOS for the high temperature bcc phase of Ti found in the literature $[82,83]$ are in line with the calculated phonon frequencies for bcc Ti. Since the mass of $\mathrm{Ti}$ is about a factor of 4 smaller than the one of $\mathrm{W}$ and the phonon frequencies scale with $1 / \sqrt{m}$ (where $m$ is the atomic mass), the stiffness of the bonds must decrease roughly by a factor of 2 in order to explain this observation. This is also in accordance with the difference in stiffness between $\mathrm{W}$ [84] and $\mathrm{Ti}$ [85] as apparent from the bulk and shear moduli, which are $65 \%$ and $25 \%$ lower in Ti, respectively. Second, adding Ti to pure $\mathrm{W}$, a new isolated peak at around $9 \mathrm{THz}$ appears, which is far above the highest frequency of pure Ti. This high frequency mode, which is dominated by Ti displacements, exhibits a rather sharp peak indicating that it is strongly localized on the Ti atoms. Apparently, the embedding of Ti in $\mathrm{W}$ leads to stronger bonds compared to pure $\mathrm{Ti}$, which together with the smaller mass leads to the high frequencies. With increasing Ti content the peak broadens and fills the gap to the lower frequency modes more and more, eventually leading to a continuous phonon DOS for more than $25 \% \mathrm{Ti}$. The maximum frequency, however, changes rather little up to a $\mathrm{Ti}$ concentration of $18.75 \%$ and then starts to decrease.

The impact of $\mathrm{Ti}$ content on the lower frequency peaks is more subtle: The peak at around $4 \mathrm{THz}$ is shifted to a slightly higher frequency when going from pure $\mathrm{W}$ to $6.26 \%$ $\mathrm{Ti}$, but then its frequency shows a continuous decrease with $\mathrm{Ti}$ addition. The peak around $6 \mathrm{THz}$ shows a monotonic decrease in frequency with increasing Ti content. In the low frequency region below $3 \mathrm{THz}$ the PDOS hardly changes up to $12.5 \% \mathrm{Ti}$, and then it slightly increases with Ti concentration.

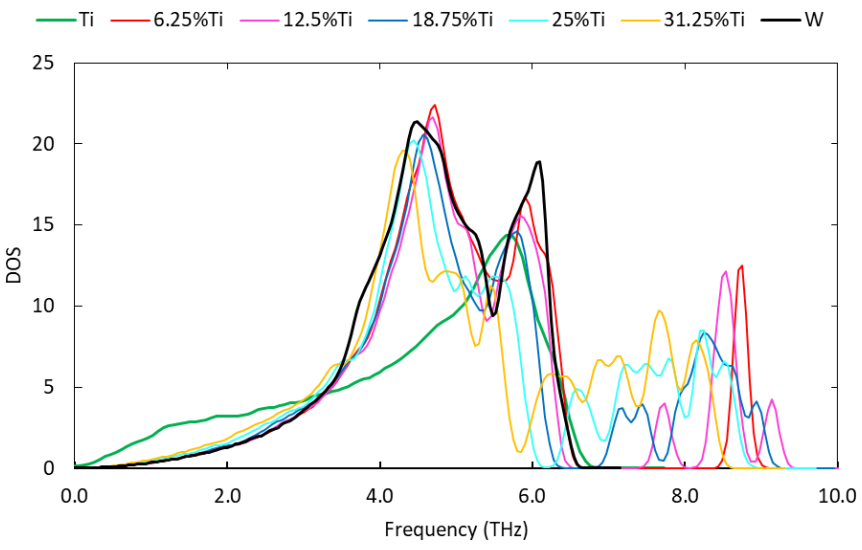

FIG. 5. Phonon density of states of WTi from 0 to $31.25 \% \mathrm{Ti}$ plus pure bcc Ti for the 16 atom supercells.

\section{E. Thermal expansion}

Figure 6 shows the unit cell volume of $\mathrm{W}_{1-x} \mathrm{Ti}_{x}$ as a function of concentration $x, V(x)$, for temperatures from 0 to $900 \mathrm{~K}$ as obtained via explicit phonon calculations using PHONOPY [43]. For all temperatures, $V(x)$ shows the same nonlinear behavior with a minimum of the atomic volume around 15 at. $\%$ Ti. The position of the minimum changes with temperature, from $18 \% \mathrm{Ti}$ at $0 \mathrm{~K}$ to $13 \% \mathrm{Ti}$ at $900 \mathrm{~K}$. This can be explained by the smaller thermal expansion of pure bcc $\mathrm{W}$ [86] compared to pure bcc Ti [64] which shifts the minimum of $V(x)$ to lower Ti concentrations as the temperature increases.

The upper panel of Fig. 7 shows the change in CTE as a function of titanium concentration. Below 12.5\%, the CTE changes only very little with Ti content. A subtle but interesting feature in this concentration range is the slope of the CTE as a function of Ti concentration: For the lowest temperature $(100 \mathrm{~K})$ it is negative, while it is close to zero at $200 \mathrm{~K}$, and then slightly increases when going to higher temperatures. For $\mathrm{Ti}$ concentrations larger than $12.5 \%$, the concentration dependence of the CTE is generally stronger and again shows a slight increase with temperature. The lower panel of Fig. 7 shows the same data in a different representation, where the CTE is plotted as a function of temperature for different $\mathrm{Ti}$ concentrations. It is also seen here that for smaller concentrations up to $12.5 \% \mathrm{Ti}$, the CTE changes only little. The largest change of CTE is found between 12.5 and $18.75 \% \mathrm{Ti}$, as is clearly visible in this figure. This may again be understood by considering the electronic DOS in Fig. 1: up to $12.5 \% \mathrm{Ti}$, the Fermi level is located in the region where the DOS is the smallest, while going above $12.5 \%$ Ti the Fermi level shifts below the pseudogap leading to weaker bonding. Moreover, the analysis of the phonon DOS in Fig. 5 showed that a smaller concentration of Ti atoms embedded in $\mathrm{W}$ show high frequency phonons due to a stronger bonding to their

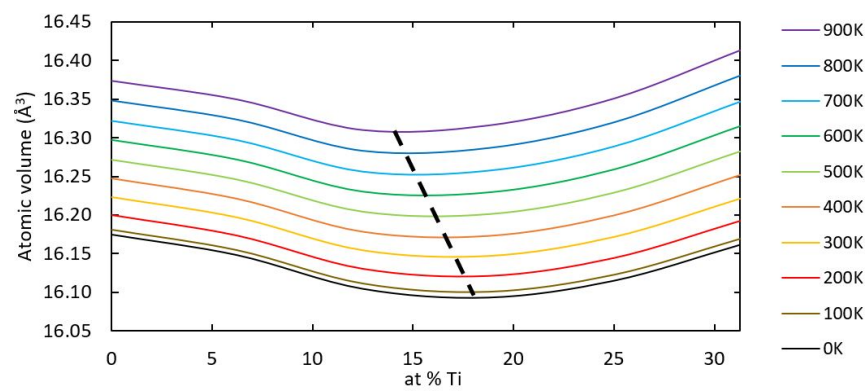

FIG. 6. Atomic volume as a function of Ti concentration for different temperatures from 0 to $900 \mathrm{~K}$. The dashed line indicates the position of the minimum for each temperature. 

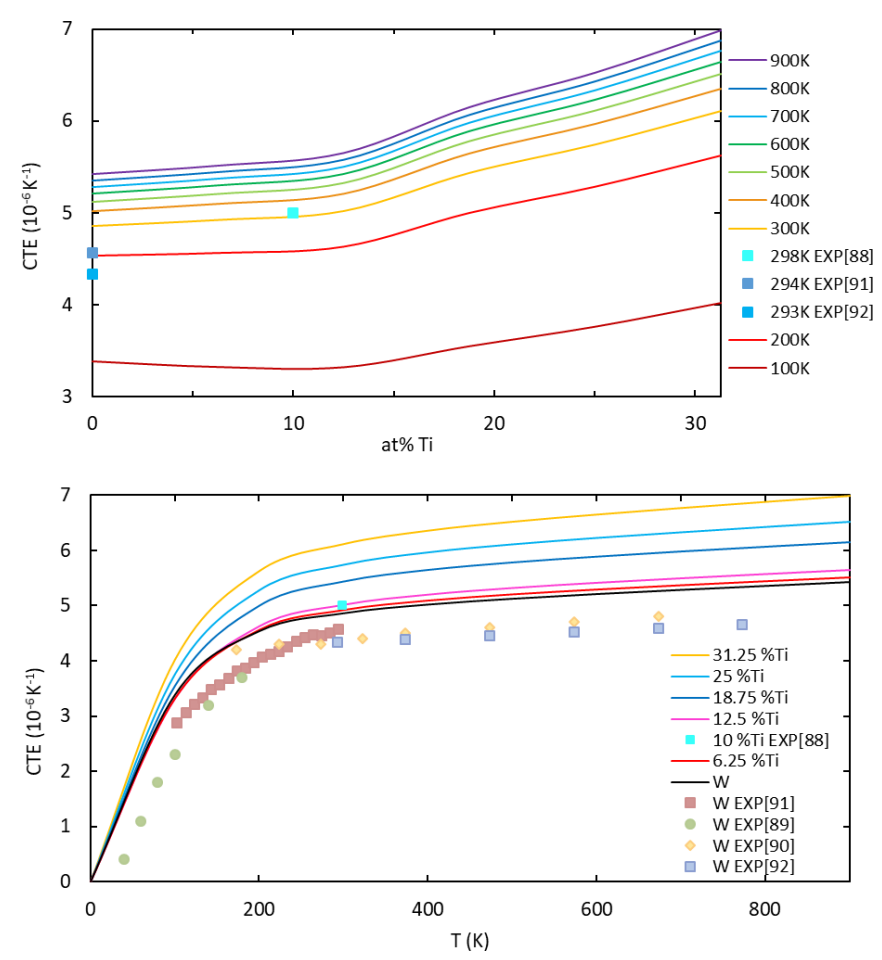

FIG. 7. Upper panel: CTE as a function of Ti concentration for different temperatures. Lower panel: CTE as a function of temperature for different $\mathrm{Ti}$ concentrations.

neighboring atoms compared to pure $\mathrm{Ti}$, while at larger $\mathrm{Ti}$ concentrations these phonon frequencies go down.

The CTE, $\alpha$, can be expressed in terms of the Grüneisen constant $\gamma$, the heat capacity $c_{v}$, and the bulk modulus $B$ via the relation $\alpha=\frac{\gamma c_{v}}{3 B}$ [87]. An analysis of the CTE in terms of these three quantities, shown in Fig. 8, gives further insight into the physical origin of the dip in the CTE below $12.5 \%$. As seen in this figure, the behavior of $\gamma, c_{v}$, and $B$ is different in three different concentration ranges. In the first range from 0 to $12.5 \% \mathrm{Ti}, \gamma$, and $c_{v}$ decrease with the $\mathrm{Ti}$ concentration, which for $T \geqslant 300 \mathrm{~K}$ is counteracted by an even stronger increase of $B$ in the denominator, leading to a slight increase of $\alpha$. For $T=100 \mathrm{~K}$, the decrease of $c_{v}$ is very pronounced, leading to a slightly negative trend of $\alpha$ as a function of Ti concentration, while for $T=200 \mathrm{~K}, \alpha$ is almost constant. From $12.5 \%$ to $18.75 \% \mathrm{Ti}$, the heat capacity and bulk modulus decrease further, while the Grüneisen parameter changes its slope and increases in this range, resulting in a stronger increase of CTE as a function of concentration. In the range from $18.75 \%$ to $31.25 \% \mathrm{Ti}$, finally, the Grüneisen parameter $\gamma$ decreases again, while the heat capacity shows a slight increase. Together with the strong decrease of $B$ this leads to a slight increase in the CTE in this concentration range. One more striking feature of Fig. 8 is the strong change of $c_{v}$ going from 100 to $200 \mathrm{~K}$, while for $T \geqslant 300 \mathrm{~K}$ the changes are much smaller. This temperature behavior of the heat capacity curve explains the large jump in CTE between 100 and $200 \mathrm{~K}$, followed by a much smaller change for each further increase in temperature.

Using the Debye-Grüneisen method to calculate the vibrational free energy is appealing in terms of easy application and
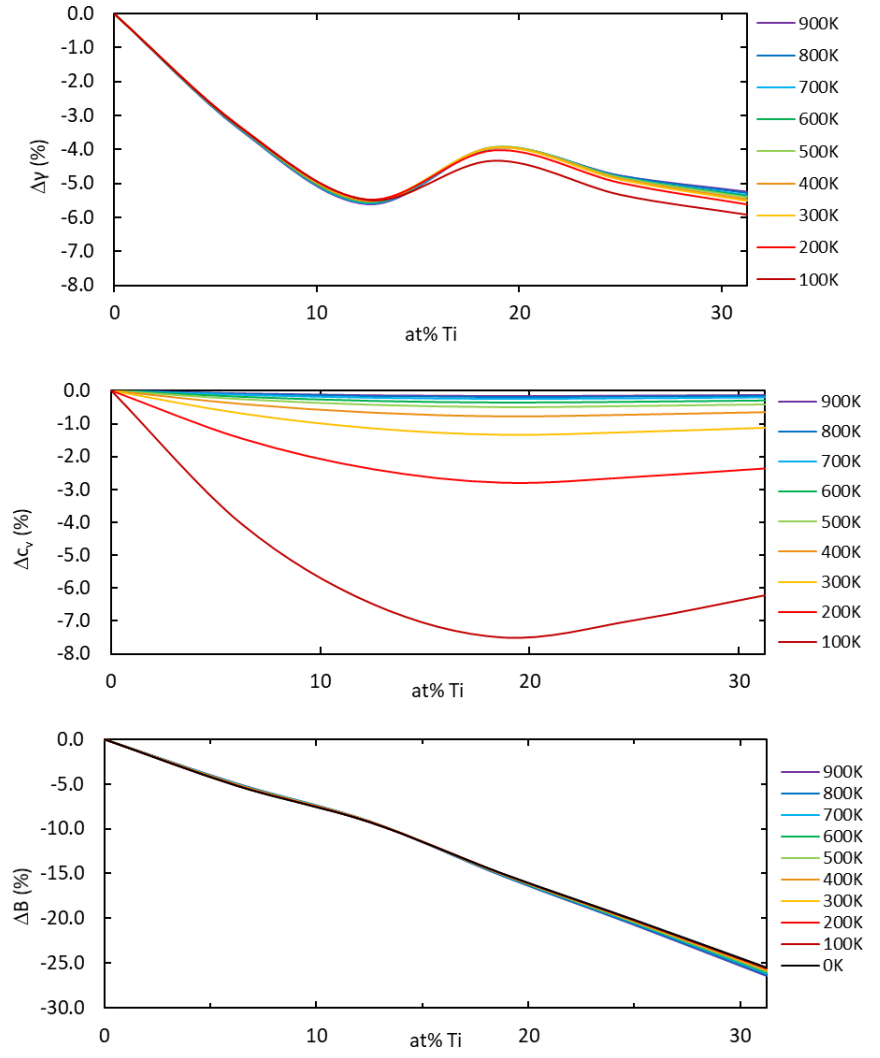

FIG. 8. Change in Grüneisen parameter $\gamma$ (top panel), heat capacity $c_{V}$ (middle panel), and bulk modulus (lower panel) relative to the value for pure $\mathrm{W}$ as a function of Ti concentration for different temperatures.

much lower computational cost compared to explicit phonon calculations. While the Debye-Grüneisen method worked in WRe only to a limited extent [23], it proved to be successful for WFe [14] and WNi [88] where it delivered results close to the experimental values. In order to understand whether it gives reasonable results for the WTi system, we show the results for the $\mathrm{CTE}$ as a function of $\mathrm{Ti}$ concentrations for different temperatures as obtained with the DG model in Fig. 9 CTE as a function of Ti concentration for different temperatures as obtained with the DG model. They fit surprisingly well to the PHONOPY results: Qualitatively all features are very well reproduced, including the decrease of CTE with Ti alloy-

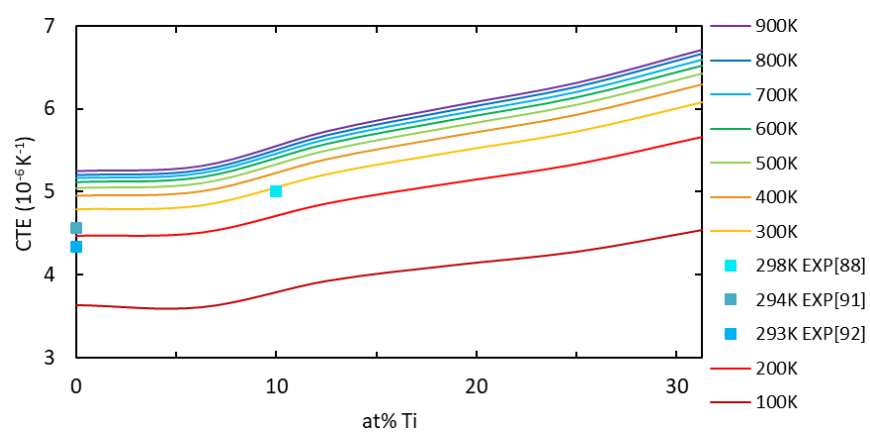

FIG. 9. CTE as a function of Ti concentration for different temperatures as obtained with the DG model. 
ing for small $\mathrm{Ti}$ concentrations at $100 \mathrm{~K}$, the steeper increase at intermediate $\mathrm{Ti}$ concentrations, and again a flatter region adding more Ti. Also quantitatively a very good agreement is found for most of the temperatures and concentrations, except for slightly smaller values for the results at $100 \mathrm{~K}$, and a shift of the steeper CTE increase to lower Ti values.

Overall the DG model allows us to predict the CTE in very reasonable agreement with PHONOPY at a fraction of the computational cost.

An experimental result of $5 \times 10^{-6} \mathrm{~K}^{-1}$ for $\mathrm{W}-10 \%$ Ti was found [89] which fits well to the calculated results. The experimental results for pure tungsten [90-93] are slightly lower than the calculated results, however such an offset between DFT and experimental results is well established [23,94].

The calculated CTE has interesting implications for residual stresses arising in layered systems used in microelectronic applications. At $300 \mathrm{~K}$ pure tungsten has a CTE between 4.3 and $4.5 \times 10^{-6} \mathrm{~K}^{-1}$ as seen in Fig. 9, while the CTE of typical metallizations are much larger, e.g., $14 \times 10^{-6} \mathrm{~K}^{-1}$ for gold [95], $16.9 \times 10^{-6} \mathrm{~K}^{-1}$ for copper [95], $19 \times 10^{-6} \mathrm{~K}^{-1}$ for silver [91], and $23.9 \times 10^{-6} \mathrm{~K}^{-1}$ for aluminum [95]. This results in a large thermal mismatch and therefore significant deformations under thermal load [96]. Adding Ti is a pathway to decrease the mismatch. However, up to $12.5 \%$ Ti the thermal mismatch does not change significantly compared to pure W. Only for larger Ti concentrations does the CTE of WTI increase substantially and close up to the values of the other metals. At $31.25 \%$, the highest concentration calculated using PHONOPY, the CTE increases by $1.25 \times 10^{-6} \mathrm{~K}^{-1}$ compared to pure $\mathrm{W}$ which in turn decreases the thermal stresses. Summarizing, the values of the CTE of WTi from Figs. 7 and 9 can be used in finite element method simulations to predict possible stresses in metal stacks arising from thermal gradients.

\section{CONCLUSION}

In this paper we investigate the electronic density of states, the concentration-dependent volume, formation energy, phonon density of states, and thermal expansion of WTi solid solutions. Analysis of the electronic DOS shows that Ti addition shifts the Fermi level out of the bcc pseudogap at around 20-25 at. \% Ti. In the investigated concentration range from 0 to $30 \% \mathrm{Ti}$, the volume generally changes only little with $\mathrm{Ti}$ content and shows a local minimum around $15 \% \mathrm{Ti}$, which can be correlated to features of the electronic structure. In this context, the EMTO-CPA calculations and the VASP supercell calculations show similar results.

The calculated formation energies show that the bcc WTi solid solution is energetically favorable at low temperature for Ti concentrations up to about 55\%. Therefore WTi has a very strong tendency to mix at low Ti concentrations. It mixes not only due to configurational entropy but already due to the $0-\mathrm{K}$ energetics of the alloy. The mixing energy with respect to bcc $\mathrm{Ti}$ and $\mathrm{W}$ is negative throughout the whole concentration range from 0 to $100 \% \mathrm{Ti}$, which is in agreement with the solid solution of WTi that forms at higher temperatures above the hcp-bcc phase transition of Ti. Finite supercell size effects change the formation energy significantly. We conclude that for situations which require highly accurate total energy calculations, such as the creation of a phase diagram, an accurate representation of the solid solution using a 128-atom SQS supercell is necessary.

The phonon density of states yields particular insight into the bonding in the WTi system: Remarkably, the phonon DOS of pure Ti and pure $\mathrm{W}$ cover the same frequency range, which is due to the fact that the lighter mass and weaker bonds in Ti compared to $\mathrm{W}$ compensate each other in terms of vibrational frequencies. Addition of $\mathrm{Ti}$ to $\mathrm{W}$ leads to new peaks in the DOS with frequencies as high as $9 \mathrm{THz}$. These new peaks, which are due to localized vibrations of Ti atoms, show that the bonding of $\mathrm{Ti}$ embedded in $\mathrm{W}$ is stronger than in pure $\mathrm{Ti}$, which together with the light mass of Ti leads to these high frequency modes. The rest of the phonon DOS undergoes rather small changes upon $\mathrm{Ti}$ addition: the phonon density slightly increases with $\mathrm{Ti}$ concentration up to $3.5 \mathrm{THz}$. For the frequency range from 3.5 to $6 \mathrm{THz}$ the peak positions generally show an increase in frequency with increasing $\mathrm{Ti}$ concentration.

The thermal expansion coefficient generally increases with Ti concentration and temperature. However, the change with titanium concentration is not linear, but decreases from 0 to $6.25 \%$ at low temperatures and then sharply increases after $12.5 \%$. When looking at the heat capacity, Grüneisen constant, and bulk modulus we can see that the decrease at lower temperatures for small concentrations is due to a decrease in Grüneisen constant and especially a strong decrease in heat capacity, while at higher temperatures the decrease in bulk modulus causes the upward trend of the CTE. For the intermediate concentration range between $12.5 \%$ and $18.75 \%$ $\mathrm{Ti}$, the Grüneisen parameter changes its slope and increases, resulting in a stronger increase of CTE as a function of concentration. The results obtained from explicit phonon calculations using PHONOPY are also compared with the ones obtained via the DG method, which is simpler to use and demands far less CPU resources. In this context a version of the DG model with two special modifications was applied which succeeded to yield results in remarkably good agreement with the PHONOPY results, not only reproducing all features qualitatively, but also showing a close match quantitatively for most of the temperatures and concentrations considered. Our work also showed good agreement between EMTO-CPA and VASP supercell calculations for the formation energy and electronic densities of states. For this reason a combined EMTO-CPA and Grüneisen approach might be of interest for similar materials and especially for multicomponent alloys including tungsten and titanium, since it delivers reasonable results at a very moderate computational cost.

\section{ACKNOWLEDGMENTS}

The authors gratefully acknowledge the financial support under the scope of the COMET program within the K2 Center "Integrated Computational Material, Process and Product Engineering (IC-MPPE)" (Project No. 859480). This program was supported by the Austrian Federal Ministries for Climate Action, Environment, Energy, Mobility, Innovation and Technology (BMK) and for Digital and Economic Affairs (BMDW), represented by the Austrian research funding association (FFG), and the federal states of Styria, Upper Austria, and Tyrol. 
[1] J. R. Lloyd, J. Clemens, and R. Snede, Copper metallization reliability, Microelectron. Reliab. 39, 1595 (1999).

[2] F. Pawlek and D. Rogalla, The electrical resistivity of silver, copper, aluminium, and zinc as a function of purity in the range 4-298 ${ }^{\circ}$ K, Cryogenics 6, 14 (1966).

[3] Y.-L. Cheng, C.-Y. Lee, and Y.-L. Huang, Copper metal for semiconductor interconnects, in Noble and Precious MetalsProperties, Nanoscale Effects and Applications (InTech, London, 2018), p. 430.

[4] S. W. Russell, S. A. Rafalski, R. L. Spreitzer, J. Li, M. Moinpour, F. Moghadam, and T. L. Alford, Enhanced adhesion of copper to dielectrics via titanium and chromium additions and sacrificial reactions, Thin Solid Films 262, 154 (1995).

[5] C.-Y.-C. Tsai, C.-C. Liu, and J.-Y. Wu, Method for enhancing adhesion between copper and silicon nitride, US Patent No. 6174793B1 (1999).

[6] S. S. Wong, C. Ryu, H. Lee, and K. W. Kwon, Barriers for copper interconnections, MRS Symp. Proc. 514, 75 (1998).

[7] J. C. Chiou, K. C. Juang, and M. C. Chen, TiW(N) as diffusion barriers between $\mathrm{Cu}$ and $\mathrm{Si}, \mathrm{J}$. Electrochem. Soc. 142, 2326 (1995).

[8] A. Baeri, V. Raineri, F. Roccaforte, F. La Via, and E. Zanetti, Study of TiW /Au thin films as metallization stack for high temperature and harsh environment devices on $6 \mathrm{H}$ Silicon Carbide, Mater. Sci. Forum 457-460, 873 (2004).

[9] C. S. Chang, T. B. Wu, C. K. Huang, W. C. Shih, and L. L. Chao, Thermal stability and oxidation resistance of W, TiW, W(N) and TiW(N) thin films deposited on Si, Jpn. J. Appl. Phys. 39, 6413 (2000).

[10] M. Fugger, M. Plappert, C. Schäffer, O. Humbel, H. Hutter, H. Danninger, and M. Nowottnick, Comparison of WTi and $\mathrm{WTi}(\mathrm{N})$ as diffusion barriers for $\mathrm{Al}$ and $\mathrm{Cu}$ metallization on $\mathrm{Si}$ with respect to thermal stability and diffusion behavior of $\mathrm{Ti}$, Microelectron. Reliab. 54, 2487 (2014).

[11] A. Kleinbichler, Adhesion of critical interfaces in microelectronics, Ph.D. thesis, Montanuniversität Leoben, 2018.

[12] T. Chookajorn and C. A. Schuh, Nanoscale segregation behavior and high-temperature stability of nanocrystalline W-20 at.\% Ti, Acta Mater. 73, 128 (2014).

[13] D. Y. Jiang, C. Y. Ouyang, and S. Q. Liu, Mechanical properties of W-Ti alloys from first-principles calculations, Fusion Eng. Des. 106, 34 (2016).

[14] Q. Q. Ren, J. L. Fan, Y. Han, and H. R. Gong, Structural, thermodynamic, mechanical, and magnetic properties of FeW system, J. Appl. Phys. 116, 093909 (2014).

[15] A. C. Lieser, C. L. Zacherl, A. Saengdeejing, Z.-K. Liu, and L. J. Kecskes, First-principles calculations and thermodynamic re-modeling of the Hf-W system, Calphad 38, 92 (2012).

[16] Z. L. Wang, W. J. Gao, Y. L. Liu, R. Li, F. S. Meng, J. P. Song, and Y. Qi, A first principles investigation of W1-xMox $(x=0-68.75$ at.\%) alloys: Structural, electronic, mechanical and thermal properties, J. Alloys Compd. 829, 154480 (2020).

[17] M. Chakraborty, J. Spitaler, P. Puschnig, and C. AmbroschDraxl, ATAT@WIEN2k: An interface for cluster expansion based on the linearized augmented planewave method, Comput. Phys. Commun. 181, 913 (2010).

[18] R. V. Chepulskii and S. Curtarolo, Calculation of solubility in titanium alloys from first principles, Acta Mater. 57, 5314 (2009).
[19] D. Nguyen-Manh, M. Muzyk, K. J. Kurzydlowski, N. L. Baluc, M. Rieth, and S. L. Dudarev, First-principles modeling of tungsten-based alloys for fusion power plant applications, Key Eng. Mater. 465, 15 (2011).

[20] M. Muzyk, D. Nguyen-Manh, J. Wróbel, K. J. Kurzydłowski, N. L. Baluc, and S. L. Dudarev, First-principles model for phase stability, radiation defects and elastic properties of W-Ta and W-V alloys, J. Nucl. Mater. 442, S680 (2013).

[21] M. Ekman, K. Persson, and G. Grimvall, Phase diagram and lattice instability in tungsten-rhenium alloys, J. Nucl. Mater. 278, 273 (2000).

[22] C. Yang and L. Qi, Ab initio calculations of ideal strength and lattice instability in W-Ta and W-Re alloys, Phys. Rev. B 97, 014107 (2018).

[23] T. Dengg, V. Razumovskiy, L. Romaner, G. Kresse, P. Puschnig, and J. Spitaler, Thermal expansion coefficient of WRe alloys from first principles, Phys. Rev. B 96, 035148 (2017).

[24] K. Masuda-Jindo, V. Van Hung, N. T. Hoa, and P. E. A Turchi, First principles calculations of thermodynamic and mechanical properties of high temperature bcc Ta-W and Mo-Ta alloys, J. Alloys Compd. 452, 127 (2008).

[25] X. Li, S. Schönecker, R. Li, X. Li, Y. Wang, J. Zhao, B. Johansson, and L. Vitos, Ab initio calculations of mechanical properties of bcc W-Re-Os random alloys: Effects of transmutation of W, J. Phys.: Condens. Matter 28, 295501 (2016).

[26] M. Ångqvist, J. M. Rahm, L. Gharaee, and P. Erhart, Structurally driven asymmetric miscibility in the phase diagram of W-Ti, Phys. Rev. Mater. 3, 073605 (2019).

[27] D. R. Marx, J. C. Turn, and J. Shi, Tungsten Titanium Targets for VLSI Device Fabrication, MRC Technical Note No 202 (1994), pp. 1-10.

[28] F. Innal, M. Legouera, F. Lekoui, and S. Mechachti, Mechanical and tribological characterization of tungsten-titanium thin film coatings deposited by dc magnetron sputtering, Appl. Math. Mech. Eng. 63, 197 (2020).

[29] V. G. Glebovsky, V. Y. Yaschak, V. V. Baranov, and E. L. Sackovich, Properties of titanium-tungsten thin films obtained by magnetron sputtering of composite cast targets, Thin Solid Films 257, 1 (1995).

[30] G. Kresse and J. Hafner, Ab initio molecular dynamics for liquid metals, Phys. Rev. B 47, 558 (1993).

[31] G. Kresse and J. Hafner, Ab initio molecular-dynamics simulation of the liquid-metal-amorphous-semiconductor transition in germanium, Phys. Rev. B 49, 14251 (1994).

[32] G. Kresse and J. Furthmüller, Efficiency of ab-initio total energy calculations for metals and semiconductors using a plane-wave basis set, Comput. Mater. Sci. 6, 15 (1996).

[33] G. Kresse and J. Furthmüller, Efficient iterative schemes for ab initio total-energy calculations using a plane-wave basis set, Phys. Rev. B 54, 11169 (1996).

[34] P. E. Blöchl, Projector augmented-wave method, Phys. Rev. B 50, 17953 (1994).

[35] G. Kresse and D. Joubert, From ultrasoft pseudopotentials to the projector augmented-wave method, Phys. Rev. B 59, 1758 (1999).

[36] J. P. Perdew, K. Burke, and M. Ernzerhof, Generalized Gradient Approximation Made Simple, Phys. Rev. Lett. 77, 3865 (1996). 
[37] J. P. Perdew, K. Burke, and M. Ernzerhof, Generalized Gradient Approximation Made Simple [Phys. Rev. Lett. 77, 3865 (1996)], Phys. Rev. Lett. 78, 1396(E) (1997).

[38] J. M. Cowley, An approximate theory of order in alloys, Phys. Rev. 77, 669 (1950).

[39] J. M. Cowley, Short-range order and long-range order parameters, Phys. Rev. 138, A1384 (1965).

[40] B. Warren, X-ray Diffraction (Dover, New York, 1990).

[41] See Supplemental Material at http://link.aps.org/supplemental/ 10.1103/PhysRevMaterials.5.043601 for short range order parameters and electronic DOS of the 16-atom SQS obtained by VASP.

[42] F. Birch, Finite elastic strain of cubic crystals, Phys. Rev. 71, 809 (1947).

[43] A. Togo and I. Tanaka, First principles phonon calculations in materials science, Scr. Mater. 108, 1 (2015).

[44] P. Debye, Zur Theorie der spezifischen wärmen, Ann. Phys. 344, 789 (1912)

[45] O. L. Anderson, A simplified method for calculating the debye temperature from elastic constants, J. Phys. Chem. Solids 24, 909 (1963).

[46] V. L. Moruzzi, J. F. Janak, and K. Schwarz, Calculated thermal properties of metals, Phys. Rev. B 37, 790 (1988).

[47] P. A. Korzhavyi, A. V. Ruban, S. I. Simak, and Y. K. Vekilov, Electronic structure, thermal, and elastic properties of Al-Li random alloys, Phys. Rev. B 49, 14229 (1994).

[48] I. A. Abrikosov, A. V. Ruban, D. Y. Kats, and Y. H. Vekilov, Electronic structure, thermodynamic and thermal properties of $\mathrm{Ni}$-Al disordered alloys from lmto-cpa-dft calculations, J. Phys.: Condens. Matter 5, 1271 (1993).

[49] VASP and PHONOPY calculation. https://phonopy.github.io/ phonopy/vasp.html.

[50] B. Grabowski, T. Hickel, and J. Neugebauer, Ab initio study of the thermodynamic properties of nonmagnetic elementary fcc metals: Exchange-correlation-related error bars and chemical trends, Phys. Rev. B 76, 024309 (2007).

[51] https://www.vasp.at/wiki/index.php/ADDGRID. Date accessed: 2020-03-26.

[52] O. K. Andersen, O. Jepsen, and G. Krier, Exact muffin-tin orbital theory, in Lectures on Methods of Electronic Structure Calculations (World Scientific, Singapore, 1995), pp. 63-124.

[53] O. K. Andersen, C. Arcangeli, R. W. Tank, T. Saha-Dasgupta, G. Krier, O. Jepsen, and I. Dasgupta, Third-Generation TBLMTO, MRS Proc. 491, 3 (1997).

[54] L. Vitos, Total-energy method based on the exact muffin-tin orbitals theory, Phys. Rev. B 64, 014107 (2001).

[55] L. Vitos, I. A. Abrikosov, and B. Johansson, Anisotropic Lattice Distortions in Random Alloys from First-Principles Theory, Phys. Rev. Lett. 87, 156401 (2001).

[56] H. Li, C. Draxl, S. Wurster, R. Pippan, and L. Romaner, Impact of $d$-band filling on the dislocation properties of bcc transition metals: The case of tantalum-tungsten alloys investigated by density-functional theory, Phys. Rev. B 95, 094114 (2017).

[57] G. D. Samolyuk, Y. N. Osetsky, and R. E. Stoller, The influence of transition metal solutes on the dislocation core structure and values of the Peierls stress and barrier in tungsten, J. Phys.: Condens. Matter 25, 025403 (2013).
[58] F. Willaime, A. Satta, M. Nastar, and O. Le Bacq, Electronic structure calculations of vacancy parameters in transition metals: Impact on the bcc self-diffusion anomaly, Int. J. Quantum Chem. 77, 927 (2000)

[59] D. Scheiber, R. Pippan, P. Puschnig, and L. Romaner, Supplementary data to $\mathrm{Ab}$ initio calculations of grain boundaries in bcc metals, Model. Simul. Mater. Sci. Eng. 24, 035013 (2016).

[60] U. Argaman, E. Eidelstein, O. Levy, and G. Makov, Thermodynamic properties of titanium from ab initio calculations, Mater. Res. Express 2, 016505 (2015).

[61] F. H. Featherston and J. R. Neighbours, Elastic constants of tantalum, tungsten, and molybdenum, Phys. Rev. 130, 1324 (1963).

[62] R. Wood, The lattice constants of high purity alpha titanium, Proc. Phys. Soc. 80, 783 (2002).

[63] P. Haas, F. Tran, and P. Blaha, Calculation of the lattice constant of solids with semilocal functionals, Phys. Rev. B 79, 085104 (2009).

[64] O. N. Senkov, B. C. Chakoumakos, J. J. Jonas, and F. H Froes, Effect of temperature and hydrogen concentration on the lattice parameter of beta titanium, Mater. Res. Bull. 36, 1431 (2001).

[65] Y. J. Hu, S. L. Shang, Y. Wang, K. A. Darling, B. G. Butler, L. J. Kecskes, and Z. K. Liu, Effects of alloying elements and temperature on the elastic properties of W-based alloys by firstprinciples calculations, J. Alloys Compd. 671, 267 (2016).

[66] L. Vegard, Die Konstitution der Mischkristalle und die Raumfüllung der Atome, Z. Phys. 5, 17 (1921).

[67] L. Vegard, XV. Die Röntgenstrahlen im Dienste der Erforschung der Materie, Z. Kristallogr. 67, 239 (1928).

[68] L. Zhang and S. Li, Empirical atom model of Vegard's law, Phys. B (Amsterdam, Neth.) 434, 38 (2014).

[69] S. Barzilai, C. Toher, S. Curtarolo, and O. Levy, Molybdenumtitanium phase diagram evaluated from ab initio calculations, Phys. Rev. Mater. 1, 023604 (2017).

[70] R. Sahara, S. Emura, S. Ii, S. Ueda, and K. Tsuchiya, Firstprinciples study of electronic structures and stability of bodycentered cubic Ti-Mo alloys by special quasirandom structures, Sci. Technol. Adv. Mater. 15, 035014 (2014).

[71] P. Erhart (private communication).

[72] J. W. D. Connolly and A. R Williams, Density-functional theory applied to phase transformations in transition-metal alloys, Phys. Rev. B 27, 5169 (1983).

[73] A. Stukowski, Visualization and analysis of atomistic simulation data with OVITO-the open visualization tool, Model. Simul. Mater. Sci. Eng. 18, 015012 (2010).

[74] J. D. Honeycutt and H. C. Andersen, Molecular dynamics study of melting and freezing of small Lennard-Jones clusters, J. Phys. Chem. 91, 4950 (1987).

[75] A. Stukowski, Structure identification methods for atomistic simulations of crystalline materials, Model. Simul. Mater. Sci. Eng. 20, 045021 (2012).

[76] A. van de Walle, Q. Hong, S. Kadkhodaei, and R. Sun The free energy of mechanically unstable phases, Nat. Commun. 6, 7559 (2015).

[77] Y. Giret, S. L. Daraszewicz, D. M. Duffy, A. L. Shluger, and K. Tanimura, Nonthermal solid-to-solid phase transitions in tungsten, Phys. Rev. B 90, 094103 (2014). 
[78] S. Jonsson, Reevaluation of the Ti-W system and prediction of the Ti-W-N phase diagram, Z. Metallkd. 87, 784 (1996).

[79] Z. Jin and C. Qiu, Thermodynamic evaluation of Ti-W system, Mater. Sci. Technol. 9, 378 (1993).

[80] O. Redlich and A. T. Kister, Algebraic representation of thermodynamic properties and the classification of solutions, Ind. Eng. Chem. 40, 345 (1948).

[81] Z.-G. Mei, S.-L. Shang, Y. Wang, and Z.-K. Liu, Densityfunctional study of the thermodynamic properties and the pressure-temperature phase diagram of Ti, Phys. Rev. B 80, 104116 (2009).

[82] H. R. Schober and P. H. Dederichs, Phonon States of Elements. Electron States and Fermi Surfaces of Alloys, Landolt-Börnstein, Group III: Condensed Matter, Vol. 13a (Springer-Verlag, Berlin, 1981).

[83] W. Petry, A. Heiming, J. Trampenau, M. Alba, C. Herzig, H. R. Schober, and G. Vogl, Phonon dispersion of the bcc phase of group-IV metals. I. bcc titanium, Phys. Rev. B 43, 10933 (1991).

[84] G. Simmons and H. Wang, Single Crystal Elastic Constants and Calculated Aggregate Properties. A Handbook (MIT Press, Cambridge, MA, 1971).

[85] M. Spittel and T. Spittel, Mechanical and physical properties of alloys and metals, in Part 3: Non-ferrous Alloys-Heavy Metals. Subvolume C: Metal Forming Data-Volume 2: Materials Group VIII, Advanced Materials and Technologies-LandoltBörnstein New Series 87-113 (Springer, Berlin, 2016), pp. 87-113.

[86] V. G. Andrianova, A. Z. Zhuk, V. Zaitchenko, E. B. Zaretskii, V. A. Petukhov, and V. Y. Chekhovskoi, Anomalous concentration-dependence of some physical-properties of tungsten rhenium alloys, Teplofiz. Vysok. Temp. 21, 80 (1983) [High Temp. 21, 70 (1983)].

[87] N. W. Ashcroft and N. D. Mermin, Solid State Physics (Holt, Rinehart and Winston, New York, 1976).

[88] S. I. Simak, A. V. Ruban, and Y. H. Vekilov, Thermodynamic, mechanical and thermal properties of Ni_W alloys from Harris functional LMTO-CPA calculations, Solid State Commun. 87, 393 (1993).

[89] K. L. Grosse, F. Xiong, S. Hong, W. P. King, and E. Pop, Direct observation of nanometer-scale Joule and Peltier effects in phase change memory devices, Appl. Phys. Lett. 102, 193503 (2013).

[90] P. Hidnert and W. T. Sweeney, Thermal expansion of tungsten, Sci. Pap. Bur. Stand. 20, 483 (1925).

[91] F. C. Nix and D. MacNair, The thermal expansion of pure metals. II: Molybdenum, palladium, silver, tantalum, tungsten, platinum, and lead, Phys. Rev. 61, 74 (1942).

[92] V. T. Deshpande and R. Pawar, X-Ray determination of the thermal expansion of tungsten, Curr. Sci. 31, 497 (1962).

[93] J. S. Shah and M. E. Straumanis, Thermal expansion of tungsten at low temperatures, J. Appl. Phys. 42, 3288 (1971).

[94] A. Debernardi, M. Alouani, and H. Dreyssé, Ab initio thermodynamics of metals: Al and W, Phys. Rev. B 63, 064305 (2001).

[95] F. C. Nix and D. MacNair, The thermal expansion of pure metals: Copper, gold, aluminum, nickel, and iron, Phys. Rev. 60, 597 (1941).

[96] G. Q. Zhang, W. D. van Driel, and X. J. Fan, Mechanics of Microelectronics (Springer, Dordrecht, Netherlands, 2006). 